\title{
Rating of Perceived Exertion as a Method of Volume Autoregulation Within a Periodized Program
}

Eric R. Helms ${ }^{\text {a }}$, Matt R. Cross ${ }^{\text {a }}$, Scott R. Brown ${ }^{\text {a }}$, Adam Storey ${ }^{\text {a }}$, John Cronin ${ }^{\text {a, b }}$, Michael C. Zourdos ${ }^{\mathrm{c}}$

${ }^{a}$ Sport Performance Research Institute New Zealand (SPRINZ), Auckland University of Technology, Auckland, New Zealand.

${ }^{\mathrm{b}}$ School of Exercise, Biomedical and Health Sciences, Edith Cowan University, Perth, Australia.

${ }^{c}$ Department of Exercise Science and Health Promotion, Muscle Physiology Laboratory, Florida Atlantic University, Boca Raton, FL.

Eric Helms

Sports Performance Research Institute New Zealand (SPRINZ), AUT University

AUT Millennium Institute

17 Antares Place, Mairangi Bay, Auckland 0632, New Zealand

Tel: (64) 021638466

E-mail: eric.helms@aut.ac.nz 


\section{$1 \quad$ Abstract}

The purpose of this investigation was to observe how a rating of perceived exertion (RPE) based autoregulation strategy impacted volume performed by powerlifters. Twelve ( $26 \pm 7 y$ rs, $n=9$ male, $n=3$ female) nationally qualified powerlifters performed the back squat, bench press and deadlift $3 \mathrm{x} / \mathrm{wk}$. on non-consecutive days in a session order of hypertrophy, power and then strength; for three weeks. Each session subjects performed an initial top set for a prescribed number of repetitions at a target RPE. A second top set was performed if the RPE score was too low, then subsequent back off sets at a reduced load were performed for the same number of repetitions. When the prescribed RPE was reached or exceeded, sets stopped; known as an 'RPE stop'. The percentage load reduction for back off sets changed weekly: there were 2, 4, or 6\% RPE stop reductions from the top set. The order in which RPE stop weeks were performed was counterbalanced among subjects. Weekly combined relative volume load (squat + bench press + deadlift), expressed as sets $\mathrm{x}$ repetitions $\mathrm{x}$ percentage one-repetition maximum was different between weeks $(\mathrm{p}<0.001)$ : $2 \%=74.6 \pm 22.3 ; 4 \%=88.4 \pm 23.8 ; 6 \%=114.4 \pm 33.4$. Combined weekly bench press volume (hypertrophy + power + strength) was significantly higher in accordance with load reduction magnitude $(2 \%>4 \%>6 \% ; \mathrm{p}<0.05)$, combined squat volume was greater in $6 \%$ vs. $2 \%(\mathrm{p}<0.05)$, and combined deadlift volume was greater in $6 \%$ vs $2 \%$ and $4 \%(\mathrm{p}<0.05)$. Therefore, it does seem that volume can be effectively autoregulated using RPE stops as a method to dictate number of sets performed.

Key Words: resistance training, autoregulation, powerlifting, rating of perceived exertion, training volume. 


\section{INTRODUCTION}

The main goal of powerlifting is to increase one-repetition maximum (1RM) in three disciplines; the back squat, bench press and deadlift. It has been well established that higher training volume (i.e. sets $\mathrm{x}$ repetitions $\mathrm{x}$ load lifted) $(21,23)$ and increased intensity (i.e. percentage of $1 \mathrm{RM})$ (19) are related to $1 \mathrm{RM}$ performance. Furthermore, when intensity progression is autoregulated week-to-week, strength progress has been greater versus a fixed progression (14). Additionally, volume autoregulation seems necessary as moderate volume was demonstrated to produce superior strength increases compared to both low and high volumes after 10 wks (5). Consequently, even though volume is related to strength performance, a point of diminishing returns seems to exist as high volume may hinder session-to-session recovery in the short term. Thus, regulating volume based on readiness and fatigue on a session-to-session basis to ensure the appropriate stimulus seems attractive.

Directly relevant to this topic, autoregulating session volume could be accomplished via measurement of average concentric velocity as it has been demonstrated that movement velocity slows in concert with diminished force production (24). Specifically, with a linear position transducer attached to the barbell $(4,22)$ a set could be terminated once velocity falls below a pre-determined threshold compared to the first or fastest repetition of the set; referred to as a 'velocity stop' $(6,12,17,24)$. Indeed, Pareja-Blanco et al. (2016) terminated each set in one group following a $40 \%$ velocity reduction and after a $20 \%$ velocity reduction in another group (18). As a result, greater muscular hypertrophy occurred in the $40 \%$ reduction group, while greater improvements in vertical jump height occurred in the $20 \%$ reduction group. Another usage of a velocity stop is to continue doing sets for a particular number of repetitions during a 
session until the last repetition of a set falls below a particular velocity threshold (i.e. an absolute number) (12), or percentage of best velocity. Thus, using either form of velocity stop can autoregulate volume to achieve desired adaptations (i.e. more volume for hypertrophy or better maintenance of velocity for power).

Although velocity stops can be used for autoregulating volume, access to linear position transducers for the individual powerlifter is limited due to cost (i.e. $>\$ 1,000$ ). Thus, using the recently established resistance training-specific rating of perceived exertion (RPE) scale $(8,30)$ may be a practical tool for volume autoregulation as no monetary cost is involved and strong inverse correlations exist between RPE and velocity with this scale in powerlifters for each discipline (squat: $r=-0.87$, bench press: $r=-0.79$, deadlift: $r=-0.82$ ) (9). Therefore, it seems that RPE could be used as a method to autoregulate volume in the absence of velocity. Indeed, using 'RPE stops' to dictate the number of sets performed was originally proposed in the powerlifting text "The Reactive Training Manual" (27). Specifically, it is proposed that an initial set can be performed for a specific number of repetitions with a target RPE for the set (i.e. 5 repetitions at 9 RPE), with subsequent sets performed with a reduced load (i.e. a 0-10\% reduction) for the same number of repetitions, until the initial RPE is reached again. It is theorized that a smaller percentage load reduction will result in fewer sets performed (i.e. RPE target is achieved with fewer sets), while a larger load reduction will result in more sets performed. These suggestions are in agreement with volume autoregulation using velocity stops (18).

Therefore, the aim of this study was to observe the impact of implementing RPE stops on training volume in powerlifters performing the back squat, bench press and deadlift in three weekly sessions; one hypertrophy-, one strength-, and one power-type training day for three weeks. Each week was assigned either a 2, 4, or 6\% RPE stop for all exercises performed that 
week. We hypothesized that volume would be greater in the 6\% RPE stop week versus the 4\% week, and the $4 \%$ week would produce more volume than the $2 \%$ week. Additionally, it was hypothesized that volume would be greatest during hypertrophy-type sessions compared to power and strength sessions.

\section{METHODS}

\section{Experimental Approach to the Problem}

In this observational study, we set out to compare the volume performed on the three powerlifting competition lifts, during different training session types over three weeks, while using three different levels of volume autoregulation. Competitive powerlifters performed the squat and bench press $3 \mathrm{x} / \mathrm{wk}$. and the deadlift $2 \mathrm{x} / \mathrm{wk}$. for three weeks in a daily undulating format. This training structure was outlined by Zourdos and colleagues (29), in which hypertrophy-, power-, and strength-type sessions were performed in that order on nonconsecutive days (i.e. Mon., Wed., Fri.). The deadlift was not performed during hypertrophytype sessions. An RPE target was provided for each exercise and subjects self-selected the load for the initial set in an attempt to hit the target RPE. For each of the three weeks a different RPE stop $(2,4$, or $6 \%)$ was employed; thus there were six possible weekly orders the RPE stop percentages could be implemented. To account for the order effect, the order of training weeks was counterbalanced across subjects. Subjects trained at their normal training facility and the investigator went to the facility to observe each subject a total of 10 times (one testing session and nine training sessions). On day 1, 72 hours prior to the first training session, subjects had anthropometrics assessed (i.e. height, and body mass) and were interviewed for further 

111 15/06).

\section{INSERT TABLE 1 HERE}

\section{Subjects}

information related to training experience, age, competitive powerlifting experience, competition results, and estimated 1RM for each discipline.

Fourteen competitive powerlifters were recruited from powerlifting clubs and gyms in the local region however, two subjects dropped out of the study prior to completion (one due to injury and one due to being unable to complete all training sessions). Thus, twelve subjects completed the protocol in full (male: $n=9$; female: $n=3$ ) (Table 1 ). The subjects had no previous experience utilizing a system of RPE-based volume autoregulation however, they were required to have at least one year of resistance training experience and meet the New Zealand national qualifying requirements for strength either in prior competition (within one year) or during testing (16). Additionally, subjects had to abide by the banned substance list of the International Powerlifting Federation (IPF) (28), fall between the age range of 18-49 years old, and be apparently healthy and free from injury or illness. Subjects were not allowed to compete during the study and were not in the midst of 'peaking' for competition at the time of data collection, which occurred between July and December. All subjects were informed of potential risks and signed an informed consent document prior to participation (University ethics approval number

\section{INSERT TABLE 1 HERE}




\section{Procedures}

One-Repetition Maximum (1RM). To establish eligibility for the study, to determine loads for warm-up sets during training days (i.e. this was done via \% of 1RM), and to familiarize each subject with the RPE scale, a 1RM test was conducted for each lift following a standardized dynamic warm-up. During testing and all training days, competition disciplines were performed in competition order (back squat, bench press, and then, deadlift) and each lift was performed in accordance with IPF regulations for movement standards and in concert with the IPF's definition of "unequipped" powerlifting (i.e. knee sleeves and weightlifting belt only) (11). To achieve the most accurate $1 \mathrm{RM}$ possible on each lift, previously validated procedures (30) were followed to aid in attempt selection. Thus, an RPE score was recorded using the resistance training-specific scale measuring repetitions in reserve (RIR) along with average concentric velocity (GymAware, Canberra, Australia) following each 1RM attempt. The warm-up sets and other specific procedures of the $1 \mathrm{RM}$ test replicated the methods described in a previous investigation (9).

Height, Body Mass and Body Mass Index (BMI). Each subject's height and body mass was assessed (Seca, model 876, Germany) by an investigator certified by the International Society for the Advancement of Kinanthropometry. Body Mass Index (BMI) was determined by the equation $B M I=\frac{\text { body-mass }(\mathrm{kg})}{(\text { height }([\mathrm{m}))]^{2}}$.

Rating of Perceived Exertion. As RIR is a more accurate measure of intensity of effort during resistance training near to failure compared to traditional RPE (7), the RIR-based RPE scale (i.e. RPE scores which correspond to RIR) (Figure 1) (30) was used throughout the study. Immediately prior to initial $1 \mathrm{RM}$ testing the RPE scale was shown to the participant and 
described in detail. Each value on the 1-10 scale was explained verbally while showing the scale to the subject. The scale was shown to subjects following every 1RM attempt, along with each warm-up set and working set on training days.

INSERT FIGURE 1 HERE

Training Protocol. After pre-testing, each subject was assigned to one of six RPE stop week orders $(2 \%, 4 \%, 6 \%$, or $4 \%, 6 \%, 2 \%$ or $4 \%, 2 \%, 6 \%$ etc.). Similar to a previous undulating powerlifting protocol (29), each day had a specific training goal: Monday: "hypertrophy", Wednesday: "power" and Friday: "strength”. Exercises performed, repetition targets, rest periods and RPE targets are displayed in Table 2.

\section{INSERT TABLE 2 HERE}

Efforts were made to ensure each subject's training occurred at the same specified time and location when possible. Occasionally rescheduling of training within the same day was necessary, but this occurred once or twice in only three subjects. On all three training days a standardized dynamic warm-up was completed followed by three warm-up sets; $42.5 \%$ 1RM for six repetitions, $60 \% 1 \mathrm{RM}$ for three repetitions, and $77.5 \% 1 \mathrm{RM}$ for a single repetition. Subjects were allowed to perform an additional warm-up prior to $42.5 \% 1 \mathrm{RM}$ if desired for a maximum of six repetitions using a lighter weight. After each warm-up set an RPE was obtained, and after all warm-up sets the investigator informed the subject of the repetition and RPE target for the day and asked the subject to select a load they believed would result in the target RPE occurring. Consultation of prior training data was allowed to assist in load selection.

Following a 3-minute rest period, the subject performed the first, or 'top' working set (TS1). If the RPE score was lower than the goal RPE on TS1, then a $2^{\text {nd }}$ top set (TS2) was performed with an adjusted load (i.e. $+2 \%$ load for every 0.5 RPE lower than the goal RPE) after 
a 3-minute rest period. The $2 \%$ load correction value was predetermined in pilot testing. If the RPE score was reached with TS1, TS2 was not performed. Likewise, if the RPE score exceeded the goal for the day, TS2 was not performed. Two top sets was the maximum, after which back off sets commenced, even if the goal RPE was not reached.

Following top set(s), a 3-minute rest period was adhered to, and "back off" sets commenced with a load modified based on the RPE stop percentage for the given week. If the RPE goal was achieved during the top set(s), the back off set load was calculated by reducing the top set load by the RPE stop percentage for the week $(98,96$ or $94 \%$ of the top set load was used for the 2,4 , and $6 \%$ weeks respectively). If the goal RPE was not reached during a top set, the load percentage reduction was applied to a hypothetical load that should have resulted in the goal RPE. The hypothetical load was also calculated by using a $2 \%$ increase or decrease for every 0.5 RPE score above or below the goal value. For example, if during the 4\% RPE stop week an 8.5 RPE was recorded at $100 \mathrm{~kg}$ for TS1 when the goal RPE was 8, top sets would conclude and a hypothetical load of $98 \mathrm{~kg}$ would be calculated. At this point, back off sets would begin with $94 \mathrm{~kg}$ as the $4 \%$ RPE stop percentage would be applied to the hypothetical load of $98 \mathrm{~kg}$ (loads for all sets are rounded to the nearest $\mathrm{kg}$ ). In the case where a repetition was failed on a top set (i.e. seven repetitions successfully completed when the goal was eight), the number of repetitions completed successfully was determined as a 10 RPE, and each missed repetition resulted in a $4 \%$ load reduction (as a full repetition is equal to a full RPE score) in calculating the hypothetical load. Thus, if the goal was eight repetitions at an 8 RPE, performing seven repetitions and failing the eighth would result in a hypothetical 8 RPE load calculated at $88 \%$ of the load used (a $12 \%$ reduction; $4 \%$ reduction for the missed repetition and an $8 \%$ reduction for the 10 RPE score being four 0.5 increments above the target RPE). Likewise, if RPE fell short of the goal even 
after TS2, a higher hypothetical load at the goal RPE was determined and back off sets were calculated from this hypothetical value. A flow chart showing how top and back off set loads were determined is shown below in Figure 2.

INSERT FIGURE 2 HERE

After each back off set, an RPE score was obtained and a 3-minute rest period was adhered to. Then, back off sets continued until an RPE equal to or greater than the target RPE was achieved. If an RPE equal to or greater than the target RPE was reported (or if not all repetitions could be completed on a back off set), the specific exercise was ceased for the day; then, a 5-minute rest period occurred prior to the next exercise, or the session concluded if it was following the deadlift. Thus, a minimum of two working sets were always performed (at least TS1 and at least one back off set if the target RPE was reached or exceeded on the first back off set). The number of back off sets was capped at eight to prevent excessive time cost to the investigators, the subjects and to retain ecological validity. The same protocol for load assignment, as outlined above, was used for all three exercises (squat, bench press, and deadlift).

\section{Statistical Analyses}

To express volume load differences in a group of powerlifters with heterogeneous strength levels, volume load was calculated relative to pre testing 1RM values (sets x reps x \% 1RM). Thus, 'relative volume load' was calculated for each subject, for each exercise (back squat, bench press, and deadlift), for the combined lifts (squat, bench press and deadlift volume summed), on each day of training (hypertrophy, power, and strength), and for each RPE stop week (2, 4, and 6\%). Means and standard deviations (SD) for relative volume load for all conditions were calculated. 
We used generalized linear mixed modelling using normal distributions with identity logit links and unstructured covariance to estimate the differences in outcome variables, while adjusting for random effects. Specifically, the model estimated the differences in the following

211 repeated conditions: 1) differences in relative volume load for the back squat, bench press, and 212 deadlift within the same week for different days (hypertrophy, power or strength); and, 2) 213 differences in relative volume load for the back squat, bench press, deadlift and combined lift 214 volume between RPE stop weeks (2, 4 or $6 \%$ ). This particular type of mixed models analysis 215 allows for the assessment of repeated effects while accounting for individual subject variance 216 and the inclusion of missing values. Bonferroni post-hoc adjustments were used for pairwise 217 comparisons, with the alpha level for significance set at 0.05 . Analysis was performed using a 218 statistical software package (IBM SPSS Statistics 21, SPSS Inc., Chicago, IL). To report the magnitude of the differences of the volumes performed, between group effect sizes (ES) were calculated for each comparison, such that the difference between means were divided by the pooled SD of each variable (2). Threshold values of $0.20,0.60,1.20$ and 2.00 were used to represent small (and the smallest worthwhile, non-trivial difference), moderate, large, and very 223 large effects (1).

\section{RESULTS}

Table 3 displays the relative volume performed on each lift, for each training goal, for all

227 three RPE stop weeks. Specific differences between, and within each RPE stop week for each lift 228 follow with p values and ES listed in text. 


\section{Back Squat: RPE Stop Comparisons}

For hypertrophy sessions, the $2 \%$ week did not produce significantly greater volume compared to either the $4 \%(\mathrm{p}=0.278)$ or $6 \%$ weeks $(\mathrm{p}=0.169)$; however, ES revealed a small difference with more volume in $2 \%$ vs. $4 \%(\mathrm{ES}=0.37)$ and $6 \%$ vs. $2 \%$ weeks $(\mathrm{ES}=0.43)$. However, the back squat volume produced on the hypertrophy session during the $6 \%$ RPE stop week was significantly higher than the volume during the $4 \%$ RPE stop week $(p=0.007$, ES $=$ 0.88). For power sessions, back squat volume increased linearly as RPE stop percentage increased. These moderate and large differences were significant $(p<0.001$ to $p=0.002, E S=0.81$ to 1.28 ) except between the $6 \%$ vs $4 \%$ RPE stop week, in which case the difference approached significance $(\mathrm{p}=0.061)$ with $6 \%$ producing moderately more volume than $4 \%(\mathrm{ES}=0.68)$. For strength sessions, more back squat volume was performed during both the $6 \%$ RPE stop week $(\mathrm{p}=0.001, \mathrm{ES}=0.87)$ and the 4\% RPE stop week ( $\mathrm{p}=0.049, \mathrm{ES}=0.56)$ compared to the $2 \% \mathrm{RPE}$ stop week. However, the difference between the back squat volume performed on strength sessions during the 4\% and 6\% RPE stop weeks was not significant $(\mathrm{p}=0.420)$ and while higher during the $6 \%$ vs $4 \%$ week, the difference was trivial ( $E S=0.15)$. When combining hypertrophy, power and strength sessions, mean back squat volume increased as RPE stop percentage increased. However, only the difference between the $6 \%$ vs $2 \%$ RPE stop weeks reached significance $(\mathrm{p}=0.011, \mathrm{ES}=0.90)$. The difference between the $6 \%$ vs $4 \%$ RPE stop weeks approached significance and was moderately higher during 6\% ( $\mathrm{p}=0.090, \mathrm{ES}=0.62)$. Finally, while the difference between the $4 \%$ and 2\% RPE stop weeks did not reach significance $(\mathrm{p}=0.239), \mathrm{ES}$ analysis revealed a small difference with more volume performed during $4 \%$ vs $2 \%$ week $(\mathrm{ES}=0.35)$. 


\section{Bench Press: RPE Stop Comparisons}

For hypertrophy sessions, there was statistically similar volume when comparing $2 \%$ and 4\% RPE stop weeks $(\mathrm{p}=0.801)$, with the $4 \%$ week's volume being only trivially greater $(\mathrm{ES}=$ 0.08). Differences in volume performed for hypertrophy sessions between the $2 \%$ and $6 \%$ RPE stop weeks $(\mathrm{p}=0.485)$ and the $4 \%$ and $6 \%$ RPE stop weeks $(\mathrm{p}=0.530)$ did not reach significance. However, ES revealed a small difference with more volume in $6 \%$ vs. $2 \%(\mathrm{ES}=0.54)$ and $6 \%$ vs. $4 \%$ weeks $(\mathrm{ES}=0.41)$. During power sessions, more volume was performed with the bench press during the $4 \%$ and $6 \%$ RPE stop weeks compared to the $2 \%$ RPE stop week ( $\mathrm{p}<0.001)$ and the magnitude of these differences were large and very large, respectively ( $E S=1.30$ to 2.42). The greater amount of volume performed with bench press on power sessions during the $6 \%$ vs. $4 \%$ RPE stop week approached significance $(\mathrm{p}=0.067)$ and was moderately higher $(\mathrm{ES}=0.70)$. For strength sessions, volume increased linearly with the bench press when comparing $4 \%$ vs. $2 \%$ RPE stop weeks ( $\mathrm{p}=0.018, \mathrm{ES}=0.96), 6 \%$ vs. $4 \%(\mathrm{p}=0.008, \mathrm{ES}=1.15)$ and $6 \%$ vs. $2 \%(\mathrm{p}<0.001$, $\mathrm{ES}=2.21$ ). When combining hypertrophy, power and strength sessions, the relationship of increasing bench press volume as RPE stop percentage increased, was statistically significant and moderate to large among weeks ( $\mathrm{p}<0.001$ to $\mathrm{p}=0.014, \mathrm{ES}=0.98$ to 1.96 ).

\section{Deadlift: RPE Stop Comparisons}

For power sessions, participants performed significantly more volume during the $6 \%$ RPE stop week vs. $2 \%(\mathrm{p}=0.009, \mathrm{ES}=1.05)$ and $4 \%$ RPE stop weeks ( $\mathrm{p}=0.002, \mathrm{ES}=1.09)$. However, there were not significant differences between the volume performed with the deadlift on power sessions during the $2 \%$ and $4 \%$ RPE stop weeks $(\mathrm{p}=0.814)$. While mean volume was greater during the $4 \%$ vs $2 \%$ week, the difference was trivial (ES=0.08). During strength 
sessions, participants performed significantly more volume during the $6 \%$ RPE stop week compared to the $2 \%$ RPE stop week ( $\mathrm{p}=0.017, \mathrm{ES}=1.05)$. The differences between the $2 \%$ and 4\% RPE stop weeks $(\mathrm{p}=0.274)$ and the $4 \%$ and 6\% RPE stop weeks $(\mathrm{p}=0.131)$ did not reach significance. However ES analysis revealed a small and moderate difference respectively, with more volume in $4 \%$ vs. $2 \%(\mathrm{ES}=0.34)$ and $6 \%$ vs. $4 \%$ weeks $(\mathrm{ES}=0.63)$. When combining power and strength sessions, more volume was performed with the deadlift during the $6 \%$ RPE stop week compared to both the $4 \%(p=0.002, E S=1.03)$ and the $2 \%$ RPE stop weeks $(p<0.001$, $\mathrm{ES}=1.32$ ). However, the aggregate deadlift volume difference between the $2 \%$ and $4 \%$ RPE stop weeks was not statistically significant $(\mathrm{p}=0.452)$; yet, ES analysis revealed a small difference with more volume performed in the $4 \%$ vs $2 \%$ week ( $E S=0.22)$.

\section{Combined Lift Volume: RPE Stop Comparisons}

When combining all volume performed with the back squat, bench press and deadlift from hypertrophy, power and strength sessions, within the same RPE stop week, volume increased linearly with RPE stop percentage. Thus, there was a significant difference in volume among all three weeks $(\mathrm{p}<0.001)$. The magnitude of the difference in total combined volume during the $4 \%$ vs $2 \%$ RPE stop week was moderate $(\mathrm{ES}=0.60)$, as was the difference between the $6 \%$ vs $4 \%$ RPE stop week (ES=0.94). Finally, there was a large difference in total combined volume comparing the $6 \%$ vs $2 \%$ RPE stop week (ES=1.48). Comparisons for the back squat, bench press, and combined lift volume for each RPE stop week are displayed, along with individual data delineated by sex, in figure 3 .

\section{INSERT FIGURE 3 HERE}




\section{Back Squat: Training Session Differences Within Week}

When comparing sessions (hypertrophy, power and strength) within each RPE stop week, back squat volume was greater on hypertrophy sessions than on power or strength sessions during the $2 \%(\mathrm{p}<0.001, \mathrm{ES}=1.93$ to 1.95$), 4 \%(\mathrm{p}<0.001$ to $\mathrm{p}=0.001, \mathrm{ES}=1.00$ to 1.58$)$ and $6 \%$ RPE stop weeks ( $\mathrm{p}<0.001, \mathrm{ES}=1.11$ to 1.44 ). The differences in back squat volume performed on power sessions relative to strength sessions within each week did not approach or reach significance during the $2 \%(p=0.598), 4 \%(p=0.805)$ or $6 \%$ RPE stop weeks $(p=0.211)$. However, ES revealed a small difference, with more volume performed during power vs strength during the $6 \%$ week $(\mathrm{ES}=0.35)$.

Bench Press: Training Session Differences Within Week

When comparing training sessions within each RPE stop week, bench press volume was greater during the hypertrophy session than both the strength and power session during the $2 \%$ RPE stop week ( $p<0.001, E S=2.20$ to 2.70 ). Bench press volume was not significantly higher $(\mathrm{p}=0.424)$ for the strength compared to the power session during the $2 \%$ RPE stop week. However, ES analysis revealed a small difference, with more volume performed during strength vs power during the $2 \%$ week $(\mathrm{ES}=0.30)$. During the $4 \%$ RPE stop week bench press volume was greater for the hypertrophy session than the strength session ( $\mathrm{p}=0.044, \mathrm{ES}=0.93$ ). However, the hypertrophy session was not significantly different from the power session during the $4 \%$ week ( $\mathrm{p}=0.111)$; yet ES analysis revealed a moderate difference with more volume performed during hypertrophy $(\mathrm{ES}=0.72)$. While not significant $(\mathrm{p}=0.431)$, there was small difference in 
323 volume performed favoring the power session when compared to the strength session during the

324 4\% RPE stop week (ES=0.29). During the 6\% RPE stop week bench press volume differences

325 between hypertrophy, power and strength sessions did not approach or reach significance 326 ( $\mathrm{p}=0.219$ to 0.659$)$. However, ES analysis revealed a moderate difference in volume favoring 327 hypertrophy $(\mathrm{ES}=0.80)$, as well as strength $(\mathrm{ES}=0.69)$ compared to the power session. The 328 volume performed on hypertrophy was trivially higher compared to the strength session $329(\mathrm{ES}=0.17)$ during the $6 \%$ week.

\section{Deadlift: Training Session Differences Within Week}

Comparing power and strength sessions, deadlift volume was similar among the $2 \%$ $(\mathrm{p}=0.649), 4 \%(\mathrm{p}=0.772)$ and the 6\% RPE stop weeks $(\mathrm{p}=0.794)$. The magnitude of these differences in volume for power sessions relative to strength sessions was trivial $(\mathrm{ES}=-0.09$ to $0.15)$ in all RPE stop weeks.

\section{DISCUSSION}

The purpose of this study was to examine the magnitude of volume performed with various RPE stop percentages. Our hypothesis was supported in that combined lift volume (sum of squat, bench press and deadlift volume) was greater during higher RPE stop percentages

341 (figure 3, panel 4). However, regarding session-type our hypothesis was only partially supported.

342 Specifically, volume during squat hypertrophy sessions was highest compared to power and 343 strength sessions during all weeks; however, hypertrophy session bench press volume was only 344 significantly greater than both power and strength volume $(\mathrm{p}<0.001, \mathrm{ES}=0.93)$ in the $2 \% \mathrm{RPE}$ 345 stop week. During the 4\% stop week hypertrophy session bench volume was significantly greater 
than strength $(\mathrm{p}=0.044)$, but not power session volume $(\mathrm{p}=0.111$, $\mathrm{ES}=0.72)$; while no significant differences between session volume for bench press existed in the $6 \%$ week $(p>$ 0.05). Furthermore, no significant differences existed in any week for session-type deadlift volume $(\mathrm{p}>0.05)$. Overall, it appears that the RPE stop system effectively produces increased volume with higher percentages stops (i.e. 6 vs. 4 vs. $2 \%$ ), however volume distribution between session-type is variable.

To illustrate the unexpected variability of volume distribution, back squat volume in strength sessions during $4 \%$ and $6 \%$ weeks was similar $(9.3 \pm 6.1$ vs $10.1 \pm 4.5 ; p=0.420)$, as was deadlift volume in power sessions during $2 \%$ and $4 \%$ weeks $(7.5 \pm 4.1$ vs $7.8 \pm 3.3$; p = $0.814)$ and bench press volume in hypertrophy sessions during $2 \%$ and $4 \%$ weeks $(15.8 \pm 3.5$ vs $16.2 \pm 5.6 ; \mathrm{p}=0.801)$. Combined weekly volume followed a linear trend corresponding to the RPE stop percentage (i.e. higher volume on greater \% stops), however the distribution of this volume was more varied within each week. Specifically, only the combined bench press volume (sum of hypertrophy, power, and strength bench press volume) was significantly different between all three RPE stop percentage weeks (i.e. $6 \%>4 \%, 6 \%>2 \%$ and $4 \%>2 \%$ ), while neither the combined volume of the back squat or deadlift was significantly different between all weeks. One explanation, is that the biomechanical similarities of the back squat and deadlift caused overlapping fatigue, which impacted volume performance on each lift for the remainder

364 of a specific week. In contrast, the bench press, as the only upper body movement utilized 365 presently, was not affected by other lifts.

It is also plausible that the mixed-sex population contributed to a varied volume 367 distribution since strength performance changes during different phases of the menstrual cycle $368(20,25)$ and because there are sex-related differences in fatigability $(3,10,13,15)$. However, 
many sex related differences dissipate with increased training experience (26); thus, given only three participants were females and their experience level, it is likely that any sex-influenced difference was minor. Individual levels of relative volume load are presented in Figure 3, delineated by sex to display potential differences between males and females.

In the most similar study to the present, Pareja-Blanco et al. autoregulated volume with velocity stops (18). Specifically, Pareja-Blanco terminated each set once a repetition was completed at a velocity that had decreased by either $20 \%$ or $40 \%$ compared to the set's initial repetition; which resulted in almost $60 \%$ more total repetitions over 8 weeks in the $40 \%$ vs. $20 \%$ velocity reduction group despite training at a similar percentage 1RM (18). In the present study, total relative volume of all lifts combined, was $18.6 \%$ greater with $4 \%$ vs. $2 \%, 29.3 \%$ greater with $6 \%$ vs. $4 \%$, and $53.4 \%$ with $6 \%$ vs. $2 \%$ RPE stop percentages. Despite the RPE stop percentage increasing the same amount from $2 \%$ to $4 \%$, and $4 \%$ to $6 \%$, volume increased $\sim 10 \%$ more from $4 \%$ to $6 \%$ compared to the difference from $2 \%$ to $4 \%$. Thus, while volume is greater with higher RPE stop percentages, it does not necessarily follow a predictable pattern of increase.

One potential concern when programming resistance training is managing fatigue within the weekly design. As established by Zourdos and colleagues (29), the modified daily undulating periodization model we used places a power session between the hypertrophy and strength sessions. This order has been demonstrated to yield improved recovery and performance during a training week compared to a traditional configuration (i.e. hypertrophy, strength, and then power); (29) thus it was implemented presently. The power session had the lowest number of repetitions paired with the lowest RPE of all days (i.e. 2 repetitions at $8 \mathrm{RPE}$ ); thus most times that the maximum back off set limit was reached (i.e. 8 sets) was during the power session. This 
392

could prove problematic if too much volume is performed during power sessions so that it subverts the purpose of recovery; therefore it is possible a lower back off set limit could be implemented during power sessions to avoid this issue.

To conclude, while this system does result in an overall predictable change in training volume, it may pose problems if a coach desires to emphasize a specific lift in training. Additionally, a limitation is that this system has only been studied in competitive powerlifters. Previous research has established that the RIR-based RPE scale that this system is based on is less accurate when used by novice lifters (30). Consequently, caution should be exercised before applying these results to different populations, and particularly with less experienced lifters. Finally, future research should compare this system to a traditional system of predetermined daily volume over time for muscle performance.

\section{PRACTICAL APPLICATIONS}

Given that the overall goal of modulating training volume was achieved using RPE stop percentages, this system of volume autoregulation could be utilized to allow training volume and stress to coincide with the desired focus of a specific training block within a periodized macrocycle. For example, when an athlete is training within a high volume mesocycle an RPE stop percentage of $6-8 \%$ could be utilized to ensure enough volume is completed. Likewise, RPE goals can be applied uniformly throughout an entire phase of training versus using differing RPE goals for different days as was done in the present investigation. For example, in place of or in addition to a higher RPE stop percentage, a lower RPE goal could be used throughout a higher volume mesocycle to slow the rate of fatigue, allowing more sets to be performed. Conversely, 
during an intensity focused training block closer to competition, a lower RPE stop percentage of $2-4 \%$ could be used alongside the option of a higher RPE goal throughout the block to ensure heavier loads are lifted in an effort to peak. Even during a taper, which stipulates maintenance of intensity with reduced volume, a $0-2 \%$ RPE stop could be programmed to ensure diminished volume.

Importantly, individual fatigability should be taken into account. Some subjects in the present study indicated that the 3-minute rest period was too short during hypertrophy sessions, and that they could have completed more sets with a longer rest period. Additionally, since some individuals performed the maximum 8 back off sets during power sessions, we recommend a lower maximum allowed volume during power sessions. This prevents total volume during power sessions from becoming similar to hypertrophy or strength sessions, in order to maintain the session goal of recovery. Another potential solution would be to apply different RPE stop percentages to different days within the week instead of applying the percentage to the entire week. For example, if varying RPE stop percentages were applied within the week to the training model in the present study, a 4-6\% percentage could have been used for hypertrophy sessions, a $0-2 \%$ percentage for power sessions and a $2-4 \%$ percentage for strength sessions.

While this system is important because it has potential utility in autoregulating volume within a resistance training plan, it is currently unknown how this system would compare to a traditional model using a predetermined volume prescription. However, as it stands this system provides a practical approach to volume regulation. Thus, practitioners are encouraged to use this method (or iterations of it; for example, different RPE stop percentages) as a way of autoregulating volume within periodized training protocols. 


\section{Acknowledgements}

We wish to thank the participants for volunteering their time and energy, and also Get Strength, NorthSport Olympic Weightlifting, CrossFit East Auckland, Club Physical New Lynn and Sunny Singh and Alex Orwin for allowing access to their training facilities and assisting with recruitment.

\section{References}

1. Batterham AM and Hopkins WG. Making meaningful inferences about magnitudes. Int J Sports Physiol Perform 1: 50-57, 2006.

2. Dankel SJ, Mouser JG, Mattocks KT, Counts BR, Jessee MB, Buckner SL, Loprinzi PD, and Loenneke JP. The widespread misuse of effect sizes. J Sci Med Sport.

3. Fulco CS, Rock PB, Muza SR, Lammi E, Cymerman A, Butterfield G, Moore LG, Braun $\mathrm{B}$, and Lewis SF. Slower fatigue and faster recovery of the adductor pollicis muscle in women matched for strength with men. Acta physiologica Scandinavica 167: 233-239, 1999.

4. Garnacho-Castaño MV, López-Lastra S, and Maté-Muñoz JL. Reliability and validity assessment of a linear position transducer. Journal of Sports Science \& Medicine 14: 128$136,2015$.

5. Gonzalez-Badillo JJ, Gorostiaga EM, Arellano R, and Izquierdo M. Moderate resistance training volume produces more favorable strength gains than high or low volumes during a short-term training cycle. J Strength Cond Res 19: 689-697, 2005.

6. González-Badillo JJ, Marques MC, and Sánchez-Medina L. The importance of movement velocity as a measure to control resistance training intensity. J Hum Kinet 29A: 15-19, 2011.

7. Hackett DA, Johnson NA, Halaki M, and Chow CM. A novel scale to assess resistanceexercise effort. J Sports Sci 30: 1405-1413, 2012.

8. Helms E, Cronin J, Storey A, and Zourdos MC. Application of the repetitions in reservebased rating of perceived exertion scale for resistance training. Strength \& Conditioning Journal 38: 42-49, 2016.

9. Helms ER, Storey A, Cross MR, Brown SR, Lenetsky S, Ramsay H, Dillen C, and Zourdos MC. RPE and velocity relationships for the back squat, bench press, and deadlift in powerlifters. J Strength Cond Res 31: 292-297, 2017.

10. Hunter SK. Sex differences in human fatigability: mechanisms and insight to physiological responses. Acta physiologica (Oxford, England) 210: 768-789, 2014. 
11. http://www.powerlifting-ipf.com/rules/technical-rules.html.

12. Jovanović M and Flanagan EP. Researched applications of velocity based strength training. J Aust Strength Cond 22: 58-69, 2014.

13. Judge LW and Burke JR. The effect of recovery time on strength performance following a high-intensity bench press workout in males and females. Int J Sports Physiol Perform 5: 184-196, 2010.

14. Mann JB, Thyfault JP, Ivey PA, and Sayers SP. The effect of autoregulatory progressive resistance exercise vs. linear periodization on strength improvement in college athletes. $J$ Strength Cond Res 24: 1718-1723 2010.

15. Maughan RJ, Harmon M, Leiper JB, Sale D, and Delman A. Endurance capacity of untrained males and females in isometric and dynamic muscular contractions. European journal of applied physiology and occupational physiology 55: 395-400, 1986.

16. http://www.nzpowerlifting.co.nz/. Accessed Mar 6 /2015.

17. Padulo J, Mignogna P, Mignardi S, Tonni F, and D'Ottavio S. Effect of different pushing speeds on bench press. Int J Sports Med 33: 376-380, 2012.

18. Pareja-Blanco F, Rodriguez-Rosell D, Sanchez-Medina L, Sanchis-Moysi J, Dorado C, Mora-Custodio R, Yanez-Garcia JM, Morales-Alamo D, Perez-Suarez I, Calbet JA, and Gonzalez-Badillo JJ. Effects of velocity loss during resistance training on athletic performance, strength gains and muscle adaptations. Scand J Med Sci Sports, 2016.

19. Peterson MD, Rhea MR, and Alvar BA. Maximizing strength development in athletes: a meta-analysis to determine the dose-response relationship. J Strength Cond Res 18: 377 $382,2004$.

20. Phillips SK, Sanderson AG, Birch K, Bruce SA, and Woledge RC. Changes in maximal voluntary force of human adductor pollicis muscle during the menstrual cycle. The Journal of Physiology 496: 551-557, 1996.

21. Radaelli R, Fleck SJ, Leite T, Leite RD, Pinto RS, Fernandes L, and Simao R. Doseresponse of 1,3, and 5 sets of resistance exercise on strength, local muscular endurance, and hypertrophy. J Strength Cond Res 29: 1349-1358, 2015.

22. Randell AD, Cronin JB, Keogh JW, Gill ND, and Pedersen MC. Reliability of performance velocity for jump squats under feedback and nonfeedback conditions. $J$ Strength Cond Res 25: 3514-3518, 2011.

23. Robbins DW, Marshall PW, and McEwen M. The effect of training volume on lowerbody strength. J Strength Cond Res 26: 34-39, 2012. 
24. Sanchez-Medina L and Gonzalez-Badillo JJ. Velocity loss as an indicator of neuromuscular fatigue during resistance training. Med Sci Sports Exerc 43: 1725-1734, 2011.

25. Sarwar R, Niclos BB, and Rutherford OM. Changes in muscle strength, relaxation rate and fatiguability during the human menstrual cycle. J Physiol 493 ( Pt 1): 267-272, 1996.

26. Storey A and Smith HK. Unique aspects of competitive weightlifting: performance, training and physiology. Sports Med 42: 769-790, 2012.

27. Tuchscherer M. The Reactive Training Manual: Developing your own custom training program for powerlifting. Reactive Training Systems, 2008, pp 55-62.

28. https://wada-main-prod.s3.amazonaws.com/resources/files/wada-2016-prohibited-listsummary-of-modifications-en.pdf.

29. Zourdos MC, Jo E, Khamoui AV, Lee SR, Park BS, Ormsbee MJ, Panton LB, Contreras RJ, and Kim JS. Modified Daily Undulating Periodization Model Produces Greater Performance Than a Traditional Configuration in Powerlifters. Journal of strength and conditioning research 30: 784-791, 2016.

30. Zourdos MC, Klemp A, Dolan C, Quiles JM, Schau KA, Jo E, Helms E, Esgro B, Duncan S, Garcia Merino S, and Blanco R. Novel resistance training-specific rating of perceived exertion scale measuring repetitions in reserve. J Strength Cond Res 30: 267$275,2016$.

\section{Table and Figure Legend:}

Table 1. Descriptive characteristics of male, female and combined powerlifters.

Table 2. Training protocol overview.

Table 3. Comparisons of relative volume loads between training goals (hypertrophy, power and strength) and between RPE stops (2, 4 and 6\%) among the back squat, bench press and deadlift.

Figure 1. Resistance-exercise specific RIR-based RPE scale

Figure 2. Load selection flow chart

Figure 3. Comparisons of total relative volume loads between RPE stop weeks for the back squat, bench press and deadlift. 
Table 1. Descriptive characteristics of male, female and combined powerlifters.

\begin{tabular}{|c|c|c|c|}
\hline & Female $(n=3)$ & Male $(n=9)$ & Combined $(n=12)$ \\
\hline Body-height (m) & $1.62 \pm 0.08$ & $1.71 \pm 0.06$ & $1.69 \pm 0.08$ \\
\hline Body-mass (kg) & $59.0 \pm 5.8$ & $81.9 \pm 12.5$ & $76.2 \pm 15.0$ \\
\hline Body-mass index $\left(\mathrm{kg} / \mathrm{m}^{2}\right)$ & $22.6 \pm 1.4$ & $27.8 \pm 2.3$ & $26.5 \pm 3.1$ \\
\hline Age $(y)$ & $36.0 \pm 6.2$ & $23.0 \pm 2.5$ & $26.3 \pm 6.8$ \\
\hline Training experience $(\mathrm{y})$ & $4.6 \pm 1.6$ & $5.1 \pm 3.4$ & $5.0 \pm 2.9$ \\
\hline Relative back squat (1RM [kg] / BM [kg]) & $1.6 \pm 0.3$ & $2.4 \pm 0.3$ & $2.2 \pm 0.5$ \\
\hline
\end{tabular}

Values are mean \pm standard deviation (SD).

Relative back squat, bench press and deadlift are presented as one-repetition maximum in kilograms divided by body mass in kilograms. 
Table 2. Training protocol overview.

\begin{tabular}{l|lll}
\hline Training Goal & Hypertrophy & Power & Strength \\
\hline Exercises & Squat & Squat & Squat \\
& Bench Press & Bench Press & Bench Press \\
& - & Deadlift & Deadlift \\
Repetitions & 8 & 2 & 3 \\
RPE & 8 & 8 & 9 \\
Rest Period & 3 minutes & 3 minutes & 3 minutes \\
\hline \multicolumn{2}{l}{ RPE = Rating of Perceived Exertion }
\end{tabular}

RPE $=$ Rating of Perceived Exertion 
Table 3. Comparisons of relative volume loads between training goals (hypertrophy, power and strength) and between RPE stops (2, 4 and 6\%) among the back squat, bench press and deadlift.

\begin{tabular}{|c|c|c|c|c|c|c|c|c|c|}
\hline \multirow{2}{*}{$\begin{array}{l}\text { RPE } \\
\text { stop }\end{array}$} & \multicolumn{3}{|c|}{ Back Squat } & \multicolumn{3}{|c|}{ Bench Press } & \multicolumn{3}{|c|}{ Deadlift } \\
\hline & Hypertrophy & Power & Strength & Hypertrophy & Power & Strength & Hypertrophy & Power & Strength \\
\hline $2 \%$ & $19.8 \pm 7.4^{*}$ & $7.0 \pm 4.2^{\dagger}$ & $7.4 \pm 3.8^{* * *}, \dagger$ & $20.2 \pm 5.1^{*}$ & $8.5 \pm 4.2^{\dagger}$ & & - & $8.0 \pm 4.6$ & $7.8 \pm 2.5$ \\
\hline $6 \%$ & $23.7 \pm 8.4^{*}$ & $\begin{array}{l}13.3 \pm \\
5.3^{\dagger \dagger \dagger}\end{array}$ & $11.7 \pm 5.1^{* * *}$ & $24.6 \pm 12.0$ & $\begin{array}{l}17.0 \pm \\
2.3^{\dagger \dagger}\end{array}$ & \pm 7 & - & $\begin{array}{l}13.7 \pm \\
4.7^{\dagger \dagger \dagger}\end{array}$ & $\begin{array}{l}13.1 \pm \\
6.9^{\dagger \dagger \dagger}\end{array}$ \\
\hline
\end{tabular}

Values are presented as mean \pm standard deviation. $\mathrm{RPE}=$ rating of perceived exertion.

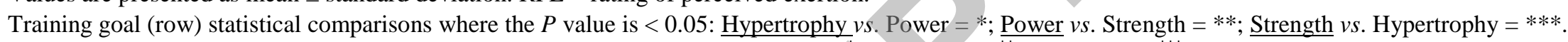

RPE stop (column) statistical comparisons where the $P$ value is $<0.05: \underline{2 \%} v s .4 \%=; \underline{4 \%} v s .6 \%={ }^{\dagger \dagger} ; \underline{6 \%} v s .2 \%={ }^{\dagger \dagger}$.

Superscript symbols, denoting statistical significance for the comparisons, are associated with the underlined metrics listed within this footnote. 
Resistance Exercise-Specific Rating of Perceived Exertion (RPE)

\begin{tabular}{c|c}
\hline Rating & Description of Perceived Exertion \\
\hline 10 & Maximum effort \\
\hline 9.5 & No further repetitions but could increase load \\
\hline 8 & 1 repetition remaining \\
\hline 8 & 2 repetitions remaining \\
\hline 7.5 & $2-3$ repetitions remaining \\
\hline $7-4$ & Little to no effort \\
\hline $5-6$ & 3 repetitions remaining \\
\hline $1-6$ & Light effort \\
\hline
\end{tabular}




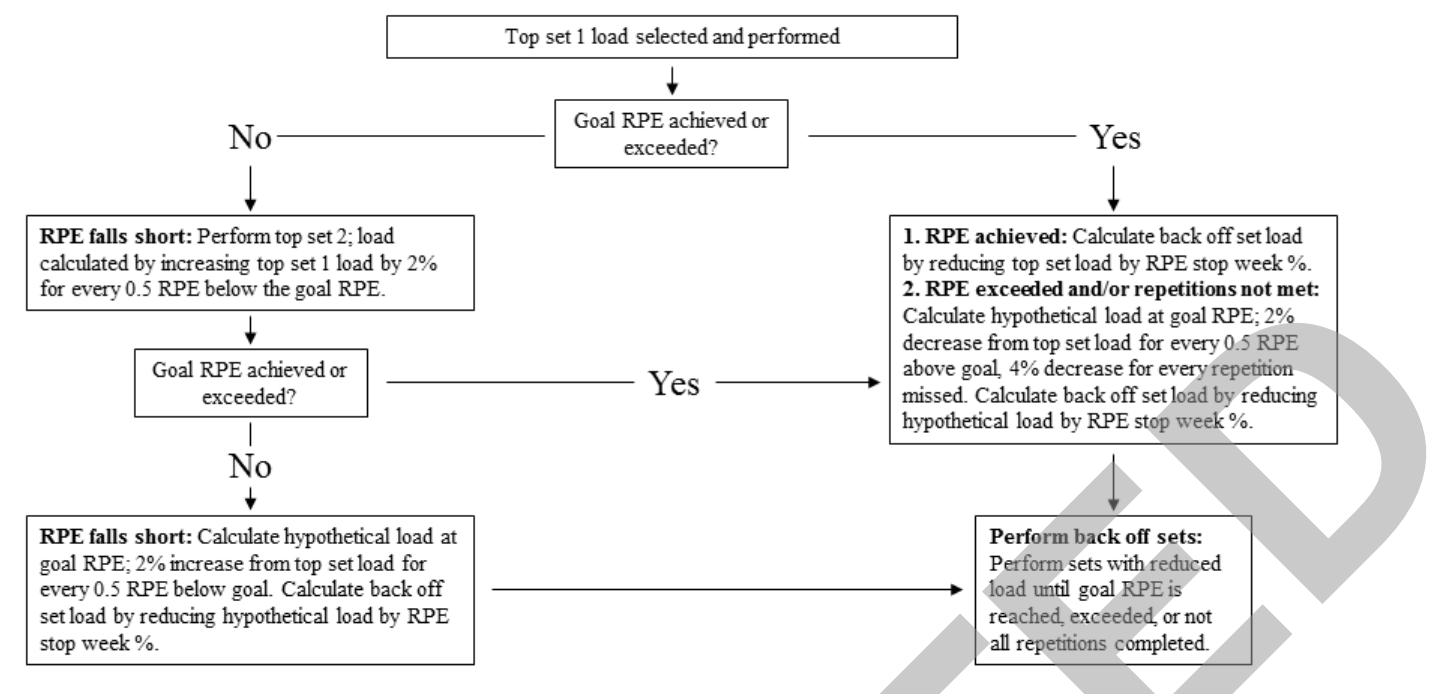

Figure 1. Flow chart of load selection for top and back off sets. 

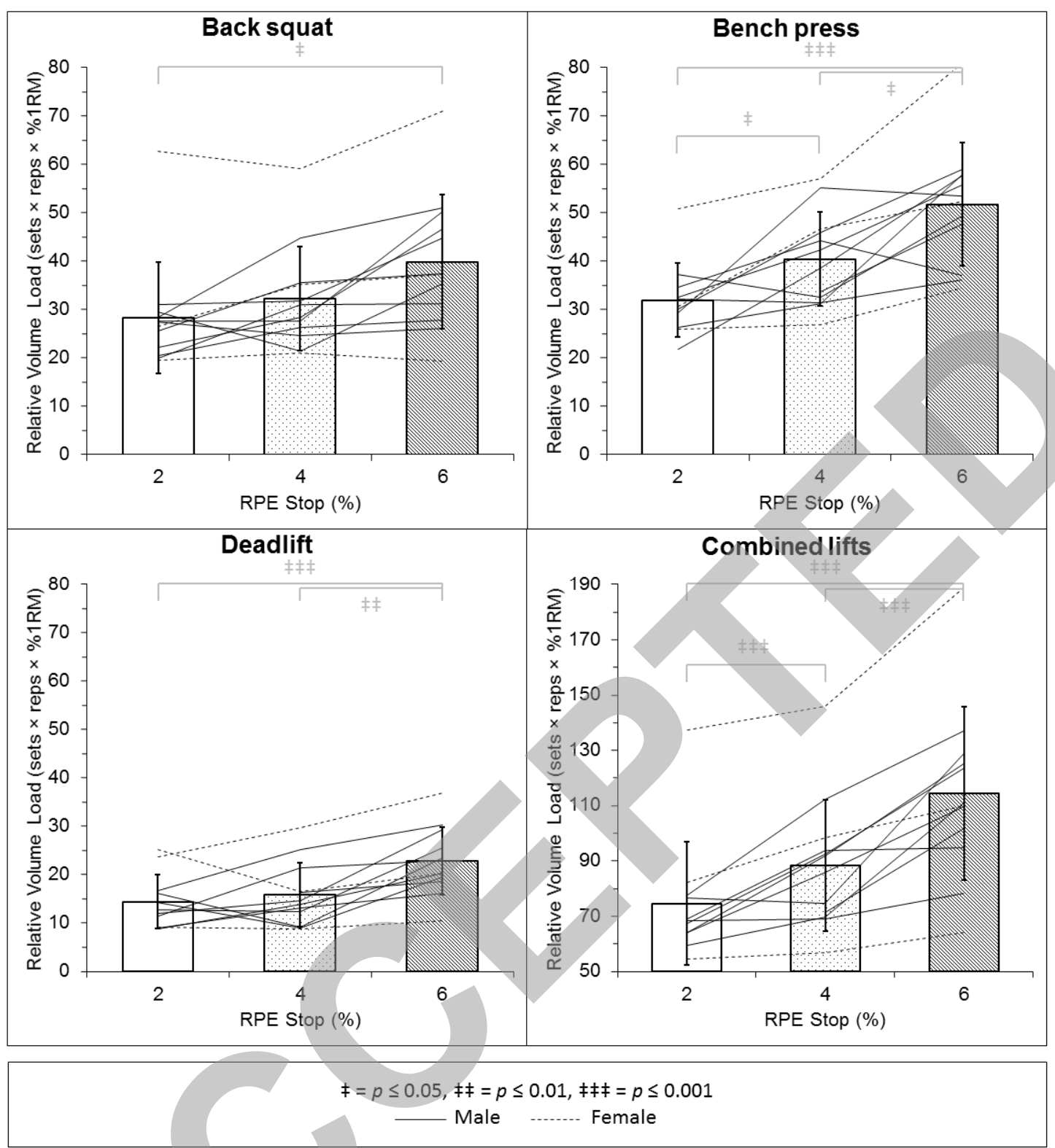